\title{
Wearable performances of lightweight PHA/PLA fiber and spun silk blended fabrics
}

\begin{abstract}
Poly hydroxyl alkanoates (PHAs) and poly lactic acid (PLA) are both environmentfriendly materials, which can be degraded into water and carbon dioxide in the environment. The PHAs and PLA are biomass polymers that can get new bio-based synthetic fiber by melt spinning. In order to integrate the good performance of the PHA/PLA composite fiber with silk, this paper took the PHA/PLA composite fiber blending with silk to fabricate a kind of new yarn by a waste silk spinning system with blending ratio of $35 / 65$, and 8 kinds of fabrics were designed and woven. The effects of the texture and the weft density of a fabric on air permeability, moisture permeability, wrinkle recovery performance and pilling resistance were investigated. The results showed that air permeability, moisture permeability and wrinkle recovery performance of blended fabrics changed from large to small in the order of satin, twill, plain, except the pilling resistance. The pilling resistance of blended fabrics improved with the increase of weft density, but air permeability, moisture permeability and wrinkle recovery performance. However, most performances of the blended fabrics were inferior to the spun silk fabric, which could be solved by improving the yarn quality, changing the fabric structure, warp and weft yarn combinations.
\end{abstract}

Keywords: poly hydroxyl alkanoate, poly lactic acid, spun silk, blended fabric, performance, spinning process technology, poly propylene, pilling resistance, biomass fibers, economic efficiency, yarn linear density, moisture permeability, blending ratio, wool abrasive, water vapour transmission
Volume I Issue 2 - 2017

\author{
Xuewei Feng,' Zhijuan Pan,,2 Sijia Di,' Ziyang \\ Chen,' Mengyao Chen' \\ 'College of Textile and Clothing Engineering, Soochow \\ University, China \\ ${ }^{2}$ National Engineering Laboratory for Modern Silk, China
}

Correspondence: Zhijuan Pan, College of Textile and Clothing Engineering Soochow University, No. 199, Renai Road, Suzhou, China, Tel +86 I3625273222, Email zhjpan@suda.edu.cn

Received: January 10, 2017| Published: March 12, 2017
Abbreviations: PHA, poly hydroxyl alkanoate; PHB, poly hydroxy butyrate; PP, poly propylene; PLA, poly lactic acid

\section{Introduction}

As the treasure in traditional textile industry of our country, silk is favored by consumers because of good skin-touch, unique pearly luster and soft hand feeling. ${ }^{1}$ But disadvantages of silk fabrics such as easy pollution, easy yellowing, easy to wrinkle, hard to care, and the high price narrow the areas of silk's applications. Therefore, it's very important to introduce new materials and technology in traditional silk industry. The design and development of the silk and new type fiber blended fabrics is worthy of further study.

In recent years, with the increasing deterioration of the environment, biomass fibers are paid more and more attention. Poly hydroxyl alkanoates (PHAs) is a class of stock which has a similar structure of carbon and energy reserves made in the bacteria. The PHAs can be degraded into water and carbon dioxide in the environment. Lemoigne M. first discovered poly-hydroxy butyrate (PHB) in $1926 .^{2}$ The performances are similar to polypropylene (PP) but biodegradable, ${ }^{3}$ commonly used in the production of daily necessities and packaging materials. As more and more novel structure and properties of PHA are found, the application scope expands to more research..$^{4-6}$

The poly lactic acid (PLA) combines the advantages of natural fibers and chemical fibers, which are biomass polymer. ${ }^{7}$ PLA fiber is very representative in the biomass synthetic fabric development. At present, PLA fiber production technology is relatively mature, ${ }^{8}$ but spinning process technology of PHAs fiber is just beginning.

Poly hydroxy alkanoates (PHAs) and poly lactic acid (PLA) are both environment-friendly materials, which can be degraded into water and carbon dioxide in the environment. Both PHAs and PLA are biomass polymers that can get new bio-based synthetic fiber by melt spinning. The composite fiber manufacturing costs are less than half of pure silk. If the composite fiber industrial production, the economic efficiency will greatly improved. In order to integrate the good performance of the PHA/PLA composite fiber with silk, this paper took the PHA/PLA composite fiber blending with silk to fabricate a kind of new yarn by a waste silk spinning system with blending ratio of 35/65 and spun silk, and 8 kinds of fabrics were designed and woven. The air permeability, moisture permeability, wrinkle recovery performance and pilling resistance of fabrics were investigated.

\section{Experimental methods}

\section{Materials}

PHA/PLA composite fiber (fiber length $50.5 \mathrm{~mm}$, linear density 1.88dtex, NingBo Tian An Biopolymer ENMAT Co., Ltd.); Spun silk, PHA/PLA composite fiber/silk blended yarn (Jiaxing Huayi Silk Co., Ltd.), yarn linear density was $120 \mathrm{Nm} / 2(83.3 \times 2$ dtex $)$, and blending ratio of blended yarn was 35/65.

\section{Yarn quality and mechanical properties of yarns}

The yarn quality and mechanical properties of blended yarn was tested by using Me100 strip tester and Instron 5967material testing machine. The results were shown in Tables $1 \& 2$ ).

\section{The structure and parameters of fabrics}

In order to integrate the good performance of the PHA/PLA composite fiber with silk, this paper took the PHA/PLA composite 
fiber blending with silk to fabricate a kind of new yarn by a waste silk spinning system with blending ratio of 35/65 [the linear density of the yarn was $120 \mathrm{Nm} / 2(83.3 \mathrm{dtex} \times 2)]$, and 8 kinds of fabrics were designed and woven.

The fabrics were divided into two series, among which $1 \#$ was spun silk fabric (control sample), 2\# 4\# were plain, twill and stain weave blended fabrics, and 5\# 8\# were twill blended fabrics which were changed the weft density. The detailed specifications and raw materials constitute of the two series of fabrics were shown in Table $3 \& 4$ respectively.

\section{Method}

Air permeability: The air permeability of fabrics was tested according to the standard GB/T 5453-1997 "Textile-Determination of air permeability" through a digital meter of air permeability (YG (B) 461E, Wenzhou-Textile Standard Instrument Factory, China). When measured, the differential pressure was $100 \mathrm{~Pa}$ and the test area was $20 \mathrm{~cm}^{2}$. Each sample tested 10 times and averaged.

Moisture permeability: The Moisture permeability was tested in accordance with standard GB/T 12704-2009 "Textiles-Determination of water vapor transmission-Permeable cup method". The test was carried out in selection of FX3150 automatic moisture permeability tester (Swiss textile company TEXTEST). Each sample tested 3 times and averaged.

Pilling resistance: The pilling resistance was tested according to the standard GB/T 4802.2-2008 "Textile-Determination of pilling performance-Part 2: modified Martindale method" using the flat abrasion instrument (YG401G, Ningbo textile Instrument Factory) and wool abrasive. Each test was 2000 times' flat abrasion and given a subjective evaluation contrast a standard sample card. Each sample tested three times then averaged. The evaluation was based on visual description as shown in Table 5 .

Wrinkle recovery performance: The crease recovery angle was tested according to the horizontal method of the standard GB/T 38191997 "Textiles-Determination of the crease recovery-angle of recovery". Sample Compression load: $(10 \pm 0.05) \mathrm{N}$; Test time: immediate elastic recovery was $(15 \pm 1) \mathrm{s}$, slow elastic recovery $5 \mathrm{~min} \pm 5 \mathrm{~s}$. Each warp and weft of the fabric were tested three times then averaged. The sum of wrinkle recovery angle of the warp and weft indicated the crease recovery of the fabric.

The weight per square meter and the covering factor of the fabrics: The weight per square meter was measured on an electronic balance (BS224S, Sartorius Ltd., Germany). The covering factor (E) of the fabrics was calculated by the following equations: ${ }^{9}$

$$
\begin{aligned}
& E_{j}=P_{j} d_{j}=y p_{j} \sqrt{T_{j}} \\
& E_{w}=P_{w} d_{w}=Y P_{w} \sqrt{T_{w}} \\
& E=E_{j}+E_{w}-E_{j} E_{w}
\end{aligned}
$$

Where $E_{j}$ and $E_{w}$ are the respectively the warp and weft covering factor of the fabrics; $\mathrm{P}_{\mathrm{j}}$ and $\mathrm{P}_{\mathrm{w}}$ are the warp and weft density (the number of yarns per $\mathrm{cm}) ; d_{j}$ and $d_{w}$ are the diameters of warp and weft yarn $(\mathrm{mm}) ; \mathrm{Y}$ is a yarn diameter coefficient; $\mathrm{T}_{\mathrm{j}}$ and $\mathrm{T}_{\mathrm{w}}$ are the warp and weft linear density (tex).

\section{Results and discussion}

\section{The air permeability of fabrics}

The air permeability values of the fabrics with different textures and weft densities were shown in Figure $1 \& 2$ ). Figure 1 showed that air permeability of blended fabrics changed from large to small in the order of satin, twill, plain. The plain weave was the lowest. Because the welt floats of plain weave were the shortest in the basic textures and the interwoven points were more than twill and stain. So the pore structure of plain weave was not as loose as twill and stain, which lead the plain weave poor air permeability. This explanation could also apply to twill and satin. Figure 1 also showed that the air permeability of spun silk fabrics was better than blended fabrics. Because the surface hairiness of PHA/PLA composite fiber/silk blended yarn was significantly higher than the spun silk, the formation of the barrier and the channel change increased, so the air permeability of blended fabrics was generally lower than the spun silk fabrics. But the air permeability of satin blended fabrics was better than plain spun silk fabrics. So when design fabrics, a more loose fabrics structure can improve the air permeability of blended fabrics.

Figure 2 showed that air permeability of blended fabrics decreased with the increase of weft density. Because the spacing between the weft yarns of the blended fabrics decreased with the increase of the welt density, which led the covering factor of fabrics improved, and the porosity decreased. Thus the air permeability decreased.

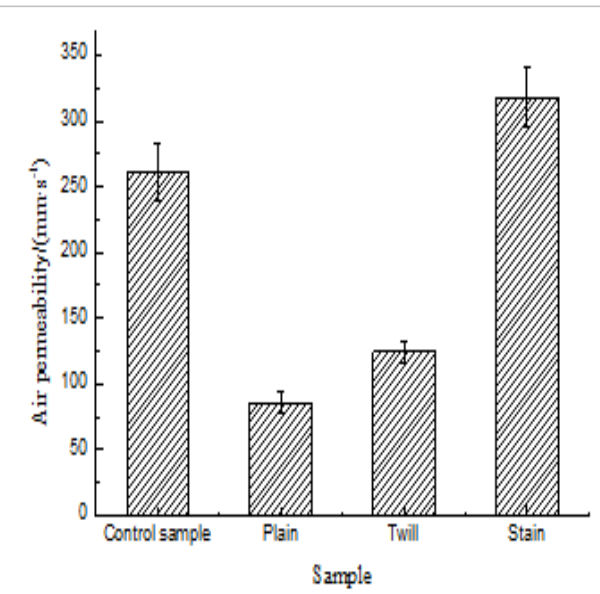

Figure I The air permeability of PHA/PLA fiber and spun silk blended fabrics with different textures.

\section{Moisture permeability}

The moisture permeability of fabrics with different textures and weft densities were shown in Figure $3 \& 4$ ). The moisture permeability is mainly affected by the fabric structure, yarn structure and raw material composition. The larger the covering factor is, the more compact the pore structure of the fabrics is, and the more unfavorable the diffusion of water vapor through the micro pores. Figure 3 showed that among different textures, the moisture permeability was little difference. The plain weave was the lowest. Because the interwoven points of plain weave were more than twill and stain. Relatively, the fabric structure was more closely. While the difference of moisture permeability between blended fabrics and spun silk fabrics was not significant. As shown in Table 3, the difference of covering factor between spun silk fabrics and blended fabrics was not significant. The 
moisture regain of PHA/PLA composite fiber was obviously lower than silk fiber. ${ }^{10}$ The moisture absorption capacity of the fibers and the moisture permeability of the final fabrics appeared to be contradictory. However, since the composite fiber had little curl and poor cohesion between the fibers, the yarns had a loose structure and a large gap between the fibers. The water vapor could pass through the space between the fibers to the lower side of the vapor pressure. Therefore, although the regain of composite fiber was far worse than the silk, the moisture permeability of blended fabrics was not affected.

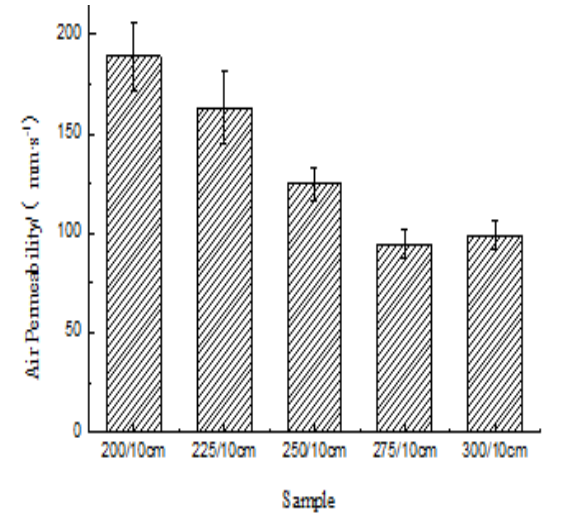

Figure $\mathbf{2}$ The air permeability of PHA/PLA fiber and spun silk blended twill fabrics with different weft densities.

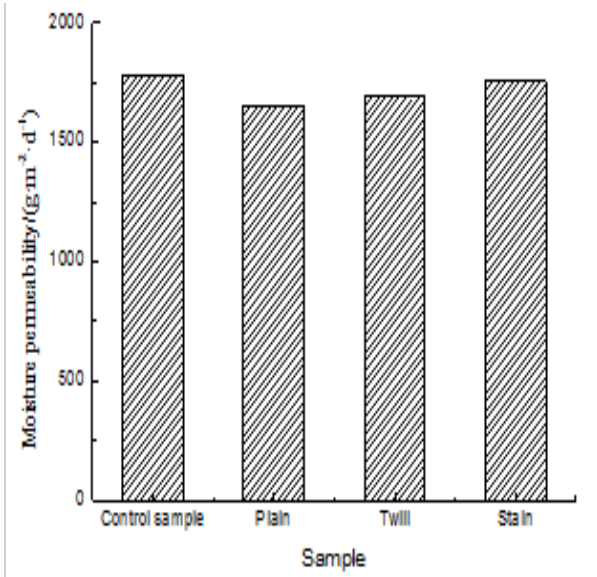

Figure 3 The moisture permeability of PHA/PLA fiber and spun silk blended fabrics with different textures.

Figure 4 showed that with the increase of the weft density, the moisture permeability of fabrics changed not significantly. Mainly because when the weft density of blended fabrics increased, there was not much difference of the covering factor (the difference between the maximum value and the minimum value was only $2.77 \%$ ), which indicated that changing the weft density of blended fabrics did not differently affect the moisture permeability. That weft density on the moisture permeability of the fabric was not significant.

\section{Pilling resistance}

The pilling resistance of fabrics with different textures and weft densities were shown in Figure $5 \&$ 6). Figure 5 showed that among different textures, the pilling resistance of blended fabrics changed from large to small in the order of plain (2\#), twill (3\#), stain (4\#). Because the interwoven points of plain weave were more than twill and stain. Fibers among the plain fabrics could be better cohesion, which was not easy to be bending and pulling. So the level of pilling resistance of $1 \#$ was the highest, the twill and stain poor. Figure 5 also showed that the pilling resistance of spun silk fabrics was better than blended fabrics. As the blended yarn quality was poor Table 1, the number of surface hairiness was higher than spun silk, which was not conductive to the improvement of pilling resistance of fabrics.

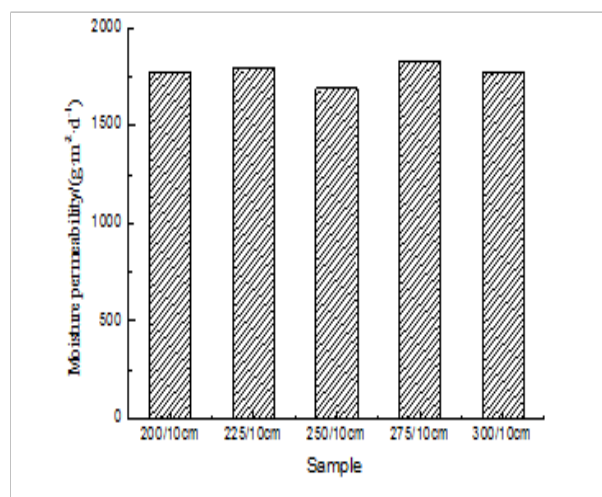

Figure 4 The moisture permeability of PHA/PLA fiber and spun silk blended twill fabrics with different weft densities.

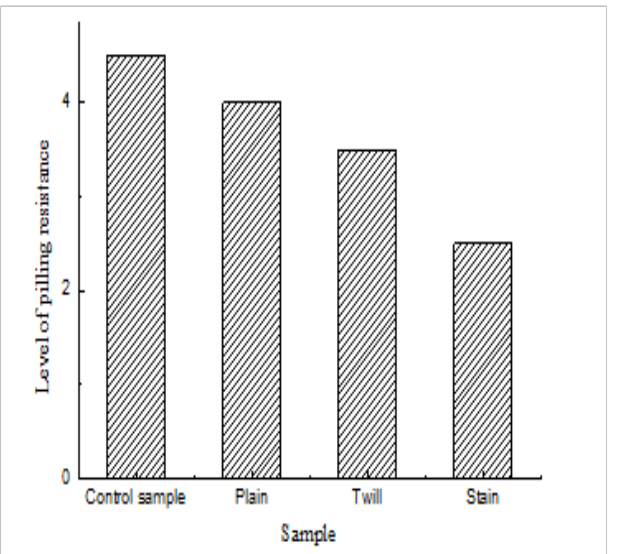

Figure $\mathbf{5}$ The pilling resistance of PHA/PLA fiber and spun silk blended fabrics with different textures.

Figure 6 showed that among the fabrics changed structural parameters, the weft density of $5 \# \sim 8 \#$ twill fabrics increased, which improved the covering factor and the cohesion of yarn and fibers. During the friction, the fibers not easily separated and exposed the surface of fabrics. So the level of pilling resistance was on the increase.

\section{Wrinkle recovery performance}

The wrinkle recovery performance of fabrics with different textures and weft densities were shown in (Figure 7) (Figure 8). Figure 7 showed that the immediate-elastic recovery angle and delayelastic recovery angle of plain (2\#) were the smallest, thus the wrinkle recovery performance of plain (2\#) was the worst. There was little difference between twill (3\#) and satin (4\#) for the delay-elastic recovery angle, respectively $335^{\circ}$ and $348^{\circ}$, and the immediate-elastic recovery angle were $279^{\circ}$ and $316^{\circ}$. The wrinkle recovery performance of blended fabrics changed from large to small in the order of stain (4\#), twill (3\#), plain (2\#), which was consistent with the general rule that the fewer interwoven points, the better wrinkle resistance. ${ }^{11}$ In addition, the wrinkle recovery performance of spun silk fabrics was better than the blended fabrics. 1\# (spun silk fabric) and 2\# (blended fabric) were plain weave fabrics. There was little difference between $1 \#$ and $2 \#$ for the immediate-elastic recovery angle, respectively $223^{\circ}$ and $204^{\circ}$. The difference of delay-elastic recovery angle was larger, respectively $325^{\circ}$ and $284^{\circ}$. The initial modulus of the fiber and yarn 
was the main factor affecting the wrinkle recovery angle of the fabrics, and it was positively related to the crease recovery. The lager initial modulus, the better the wrinkle recovery. As could be seen in Table 2, the initial modulus of spun silk was greater than the blended fabrics. So the wrinkle recovery was higher.

Figure 8 showed that among the fabrics changed structural parameters (5\# 8\# twill fabrics), the variation of the immediateelastic recovery angle and delay-elastic recovery angle showed a slight decrease with the increase of weft density. The larger the weft density, the sliding resistance among the yarn in the fabrics was larger. The yarn would be more difficult for relative movement when the external force to release, so the wrinkle recovery tended to decrease.

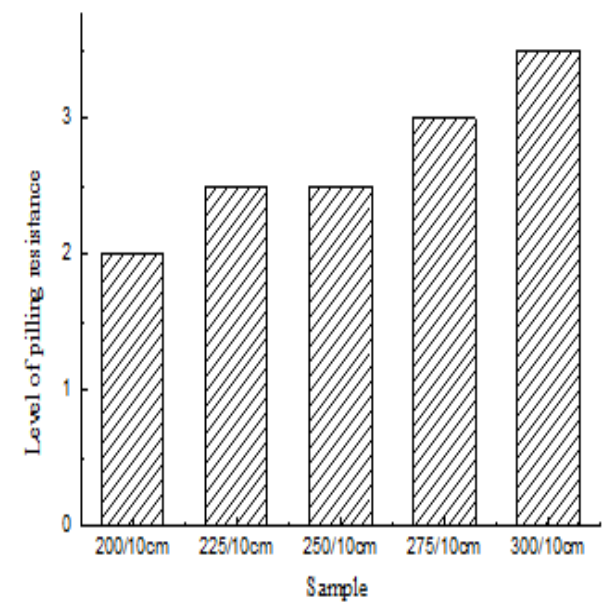

Figure 6 The pilling resistance of PHA/PLA fiber and spun silk blended twill fabrics with different weft densities.

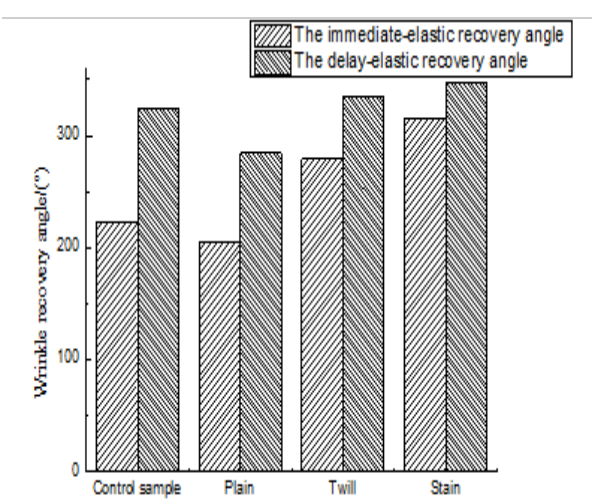

Figure 7 The wrinkle recovery performance of PHA/PLA fiber and spun silk blended fabrics with different textures.

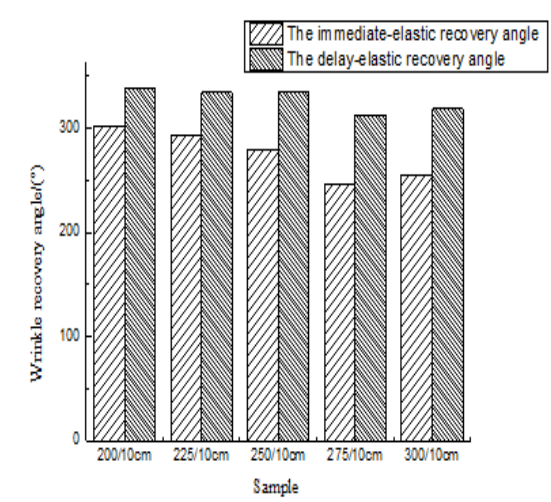

Figure 8 The wrinkle recovery performance of PHA/PLA fiber and spun silk blended twill fabrics with different weft densities.

Table I Indicators of PHA/PLA composite fiber and spun silk blended yarn and control sample

\begin{tabular}{lllll}
\hline $\begin{array}{l}\text { Yarn count and yarn } \\
\text { compositiona }\end{array}$ & $\begin{array}{l}\text { Quality uneven rate/ } \\
\text { (\%) }\end{array}$ & $\begin{array}{l}\text { Coefficient of variation of } \\
\text { quality/(\%) }\end{array}$ & $\begin{array}{l}\text { Hairiness(h)/ } \\
\text { (Pieces / I Om) }\end{array}$ & $\begin{array}{l}\text { Hairiness standard } \\
\text { deviation(sh)/ } \\
\text { (Pieces/I0m) }\end{array}$ \\
\hline $\begin{array}{l}\text { Spun Silk } \\
\begin{array}{l}\text { PHA/PLA Composite Fiber / } \\
\text { Spun silk }\end{array}\end{array}$ & 7.13 & 8.99 & 3.67 & 1.12 \\
\hline
\end{tabular}

Table 2 Mechanical properties of PHA/PLA composite fiber and spun silk blended yarn and control sample

\begin{tabular}{llll}
\hline Yarn count and yarn composition & Breaking tenacity cN/dtex) & Extension at break (\%) & Initial modulus cN/dtex) \\
\hline PHA/PLA Composite Fiber & $1.61 \pm 0.46$ & $12.63 \pm 3.38$ & $59.35 \pm 15.65$ \\
Spun Silk & $2.92 \pm 0.09$ & $7.74 \pm 0.24$ & $54.48 \pm 6.27$ \\
PHA/PLA Composite Fiber /Spun Silk & $1.89 \pm 0.15$ & $6.67 \pm 0.55$ & $45.10 \pm 4.56$ \\
\hline
\end{tabular}

Table 3 Fabric specifications of PHA/PLA fiber and spun silk blended fabrics with different textures and control sample

\begin{tabular}{lllllllll}
\hline $\begin{array}{l}\text { Sample } \\
\text { code }\end{array}$ & $\begin{array}{l}\text { Warp } \\
\text { ingredient }\end{array}$ & $\begin{array}{l}\text { Weft } \\
\text { ingredient }\end{array}$ & Texture & \multicolumn{2}{l}{$\begin{array}{l}\text { Design Density I } \\
(/ 10 \mathrm{~cm})\end{array}$} & \multicolumn{2}{l}{$\begin{array}{l}\text { Finished Density I } \\
(/ 10 \mathrm{~cm})\end{array}$} & $\begin{array}{l}\text { Covering } \\
\text { Factor (E)/ } \\
(\%)\end{array}$ \\
\cline { 5 - 8 } & & & & $\mathbf{P j}$ & $\mathbf{P w}$ & $\mathbf{P j}$ & $\mathbf{P w}$ & \\
\hline I\# & Spun Silk & Spun Silk & Plain & 540 & 250 & 572 & 271 & 85.8 \\
2\# & $35 / 65$ & $35 / 65$ & Plain & 540 & 250 & 598 & 279 & 86.7 \\
$3 \#$ & $35 / 65$ & $35 / 65$ & Twill & 540 & 250 & 603 & 303 & 88.5 \\
$4 \#$ & $35 / 65$ & $35 / 65$ & Stain & 540 & 250 & 606 & 296 & 87.9 \\
\hline
\end{tabular}

*The proportions shown in the above table are composite fiber, spun silk 
Table 4 Fabric specifications of PHA/PLA fiber and spun silk blended twill fabrics with different weft densities

\begin{tabular}{|c|c|c|c|c|c|c|c|c|}
\hline \multirow[t]{2}{*}{$\begin{array}{l}\text { Sample } \\
\text { Code }\end{array}$} & \multirow[t]{2}{*}{$\begin{array}{l}\text { Warp } \\
\text { Ingredient }\end{array}$} & \multirow[t]{2}{*}{$\begin{array}{l}\text { Weft } \\
\text { Ingredient }\end{array}$} & \multirow[t]{2}{*}{ Texture } & \multicolumn{2}{|c|}{$\begin{array}{l}\text { Design Density / } \\
(/ 10 \mathrm{~cm})\end{array}$} & \multicolumn{2}{|c|}{$\begin{array}{l}\text { Finished Density / } \\
(/ 10 \mathrm{~cm})\end{array}$} & \multirow[t]{2}{*}{$\begin{array}{l}\text { Covering Factor } \\
\text { (E)/ (\%) }\end{array}$} \\
\hline & & & & $\mathbf{P j}$ & Pw & $\mathbf{P j}$ & Pw & \\
\hline $5 \#$ & $35 / 65$ & $35 / 65$ & Twill & 540 & 200 & 606 & 305 & 87.8 \\
\hline $6 \#$ & $35 / 65$ & $35 / 65$ & Twill & 540 & 225 & 608 & 321 & 88.5 \\
\hline $7 \#$ & $35 / 65$ & $35 / 65$ & Twill & 540 & 275 & 607 & 372 & 89.8 \\
\hline 8\# & $35 / 65$ & $35 / 65$ & Twill & 540 & 300 & 606 & 416 & 90.3 \\
\hline
\end{tabular}

*The proportions shown in the above table are composite fiber, spun silk

Table 5 The evaluation form of the pilling performance

\begin{tabular}{ll}
\hline Level & Visual description \\
\hline 5 & Almost unchanged \\
4 & Slight fuzzing and (or) slight pilling \\
3 & Moderate fuzzing and (or) moderate pilling. Different sizes and densities of the ball cover part of the surface of the specimen. \\
2 & Obvious fuzzing and (or) obvious pilling. Different sizes and densities of the ball cover most of the surface of the specimen. \\
I & Serious fuzzing and (or) serious pilling. Different sizes and densities of the ball cover the entire surface of the specimen.
\end{tabular}

\section{Conclusions}

In summary, this paper took the PHA/PLA composite fiber blending with spun silk to fabricate a kind of new yarn by a waste silk spinning system with blending ratio of 35/65, and 8 kinds of fabrics were successfully designed and woven. Based on this, the effects of the texture of a fabric and the weft density on wearable performances such as air permeability, moisture permeability, wrinkle recovery performance and pilling resistance were investigated. The results showed that air permeability, moisture permeability and wrinkle recovery performance of blended fabrics changed from large to small in the order of satin, twill, plain, except the pilling resistance. The pilling resistance of blended fabrics improved with the increase of weft density, but air permeability, moisture permeability and wrinkle recovery performance. However, most wearable performances of the blended fabrics were inferior to the spun silk fabric. Mainly due to the poor quality of blended yarn, and yarn hairiness was too much. Fabrics with different textures and weft densities showed different performances. Therefore, in the future research, it should be paid more attention to improve the yarn quality, change the fabric structure, warp and weft yarn combinations to solve these problems. The quality of the blended yarn can be improved by adding nucleating agents, blending with other fibres, or by modifying the process to obtain a fabric having better performance. When design blended fabrics, a loose structure with suitable warp and weft density should be a good choice to product fabrics with excellent properties. In the future application research, the wearable performances can be improved by improving the quality of yarn, changing the fabric structure, the combination of warp and weft, etc., expecting it to get more applications in areas such as clothing, home textiles and accessories.

\section{Acknowledgments}

None.

\section{Conflict of interest}

Author declares there is no conflict of interest in publishing the article.

\section{References}

1. Wang F. Redesigning of silk garment fabrics. Silk. 2012;49(11):57-61.

2. Cuoqiang C, Daixu W. Microbial Poly hydroxy alkanoates. Beijing: Chemical Industry Press; 2014. p. 4-7.

3. Cuoqiang C. Current status and industrialization of biopolyesters poly hydroxy alkanoate (PHA). New Chemical Materials. 2010;38(10):1-7.

4. Xiangling C, Chaosheng W, Huaping W. Study on PET/PHA blend spinning research. Synthetic fiber industry. 2011;34(1):49-51.

5. Lizhen W, Yunxuan W. Research on properties of PHBV/PBS blends. Plastics Industry. 2011;39(10):91-95.

6. Jian T, Dan H, Liu Li, et al. Research on thermal performance, mechanical properties and biodegrade ability research performance of PLA, PPC and PHBV blends. Ion Exchange and Adsorption. 2010;26(1):59-67.

7. Yuan Y, Jianping, Yonggui L, et al. Performances research of PLA fiber and its blended fabric. Shanghai Textile Science \& Technology. 2008;36(2):54-56.

8. Penggang L, Fangli Z, Yan F, et al. Development and performance evaluation of poly lactic acid fabric [J]. Synthetic Fiber in China. 2015;44(8):35-37.

9. Gu Ping. Fabric weaves and structures. Shanghai: Donghua University Press; 2010. p. 179-182.

10. Xuewei F, Zhijuan P, Jigen W, et al. Structure and properties of PHA/PLA composite fiber. Modern Silk Science \& Technology. 2016;31(1):1-3,6.

11. Yu Weidong. Textile materials science. Beijing: China Textile Press; 2006. p. 318-320. 